\title{
EXACT SEQUENCES OF SPECTRA AND DUALITY
}

\author{
NICHOLAS J. KUHN ${ }^{1}$
}

\begin{abstract}
We note that duality arguments show that, under certain circumstances, an exact sequence of spectra remains exact after smashing with a finite complex. Among various applications, we show that the validity for $S^{0}$ of $\mathrm{G}$. W. Whitehead's symmetric products conjecture implies that the conjecture is "almost" true for all finite complexes.
\end{abstract}

1. Introduction. In [6], we studied sequences of spectra $\cdots \rightarrow E_{2} \rightarrow E_{1} \rightarrow E_{0}$ having the property that application of the 0 th space functor $\Omega^{\infty}$ yields a sequence of spaces of the form $\cdots \rightarrow X_{2} \times X_{1} \rightarrow X_{1} \times X_{0} \rightarrow X_{0}$, with all maps being the obvious composites of projections and inclusions. Such an exact sequence induces a long exact sequence on homotopy groups in nonnegative degrees.

In this note, we observe that a simple duality argument shows that exact sequences often remain exact after being smashed with a finite complex. Thus there is more to be gleaned from knowing that a sequence is exact than might at first be apparent.

As applications of this principle, we offer the following three examples (which will be elaborated upon in $\S 3$ ).

In [4], D. Kahn and S. Priddy showed that, localized at a prime $p$, there is a stable map $\lambda: \Sigma^{\infty} B \Sigma_{p} \rightarrow \Sigma^{\infty} S^{0}$ such that $\Omega^{\infty} \lambda: Q B \Sigma_{p} \rightarrow Q_{0} S^{0}$ is the projection onto a direct factor. Here $Q X=\Omega^{\infty} \Sigma^{\infty} X$, and $Q_{0} S^{0}$ is the component of the basepoint. Thus $\pi_{q}^{s}\left(B \Sigma_{p}\right) \rightarrow \pi_{q}^{s}\left(S^{0}\right)$ is onto for $q>0$. We use this to show that if $Y$ is a finite complex and $n=2 \operatorname{dim} Y+1$, then $\Omega^{n} Q\left(B \Sigma_{p} \wedge Y\right) \rightarrow \Omega^{n} Q Y$ is also the projection onto a direct factor. Thus $\pi_{q}^{s}\left(B \Sigma_{p} \wedge Y\right) \rightarrow \pi_{q}^{s}(Y)$ is onto for $q>2 \operatorname{dim} Y$ (this has been independently observed by J. Jones [3]).

In $[5,7]$, the Kahn-Priddy epimorphism was extended to a long exact sequence to conclude that, localized at $p$,

$$
\operatorname{ker}\left\{\pi_{q}\left(\operatorname{SP}^{p^{k}} Y\right) \rightarrow \pi_{q}\left(\operatorname{SP}^{p^{k+1}} Y\right)\right\}=\operatorname{ker}\left\{\pi_{q}\left(\operatorname{SP}^{p^{k}} Y\right) \rightarrow \pi_{q}\left(\operatorname{SP}^{\infty} Y\right)\right\}
$$

is valid when $Y=S^{0}$. Here $\mathrm{SP}^{m} E$ denotes the $m$ th symmetric product of a spectrum $E$. This was originally conjectured to be true for all $Y$ by G. W. Whitehead [9], but a counterexample was found by $\mathrm{P}$. Welcher [11] when $p=2, k=0, q=5$ and $Y=S^{0} \cup_{\nu} e^{4}$. In spite of this, we use the result for $S^{0}$ to show that if $Y$ is a finite complex, then (1.1) is true for all but a finite number of exceptional pairs $(k, q)$.

Received by the editors April 27, 1985.

1980 Mathematics Subject Classification. Primary 55P42, 55P47; Secondary 55P25, 55S15.

${ }^{1}$ Partially supported by N.S.F. 
As a final example, recall that $\mathbf{J}$. Becker showed that there is an infinite loop map $Q B O(2) \rightarrow B O$ which is the projection onto a direct factor [2]. Using this, we show that there is a similar projection $\Omega^{6} Q\left(B O(2) \wedge \mathbf{C} P^{2}\right) \rightarrow B U \times \mathbf{Z}$.

2. Exact sequences and duality. We recall some definitions from [6]. A spectrum $P$ is spacelike if it is a wedge summand of a suspension spectrum. A fibration sequence $A \rightarrow B \stackrel{f}{\rightarrow} C$ is short exact if $\Omega^{\infty} f: \Omega^{\infty} B \rightarrow \Omega^{\infty} C$ is the projection onto a direct factor, so that $\Omega^{\infty} B=\Omega^{\infty} A \times \Omega^{\infty} C$. The map $f$ is said to be onto.

A sequence of spectra $\cdots \rightarrow X_{2} \rightarrow X_{1} \rightarrow X_{0} \rightarrow E_{0}$ is exact if it is obtained from a diagram of the form

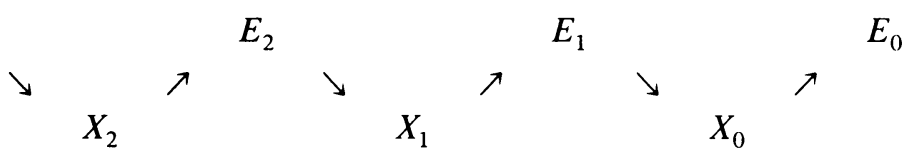

where each $E_{k+1} \rightarrow X_{k} \rightarrow E_{k}$ is short exact.

For the remainder of this paper, we will assume that all spectra are C. W. spectra of finite type (possibly localized at $p$ ). Thus any spectrum $E$ considered will be the direct limit of a sequence of finite spectra.

The following is the key observation of this paper.

THEOREM 2.1. Suppose that $\cdots \rightarrow X_{2} \rightarrow X_{1} \rightarrow X_{0} \rightarrow E_{0}$ is an exact sequence of spectra and that $\pi_{q}\left(X_{k}\right)$ is finite for $q \geqslant 0$ and $k \geqslant 1$. If $Y$ is a finite complex such that the $n$-dual of $Y$ is spacelike, then there is an exact sequence

$$
\cdots \rightarrow \Sigma^{-n} X_{2} \wedge Y \rightarrow \Sigma^{-n} X_{1} \wedge Y \rightarrow \Sigma^{-n} X_{0} \wedge Y \rightarrow \Sigma^{-n} E_{0} \wedge Y .
$$

Note that, if $Y$ is $(-1)$-connected, any $n \geqslant 2 \operatorname{dim} Y$ satisfies the conditions.

Proof. To begin, it suffices to prove the theorem with the long exact sequence replaced by a short exact sequence $A \rightarrow B \rightarrow C$ such that $\pi_{q}(A)$ is finite for $q \geqslant 0$.

Standard arguments (see [6]) using the adjoint functors $\left(\Sigma^{\infty}, \Omega^{\infty}\right)$ show that $\Sigma^{-n} A \wedge Y \rightarrow \Sigma^{-n} B \wedge Y \rightarrow \Sigma^{-n} C \wedge Y$ will be exact if and only if

$$
0 \rightarrow\left[P, \Sigma^{-n} A \wedge Y\right] \rightarrow\left[P, \Sigma^{-n} B \wedge Y\right] \rightarrow\left[P, \Sigma^{-n} C \wedge Y\right] \rightarrow 0
$$

is exact for all spacelike spectra $P$.

Recall the duality isomorphism $[X \wedge D Y, Z] \simeq[X, Z \wedge Y]$, valid if both $X$ and $Y$ are finite [1, p. 195]. Thus the exactness of $A \rightarrow B \rightarrow C$ implies that (2.2) is exact for all finite spacelike $P$, since $\Sigma^{n} P \wedge D Y$ will be spacelike.

For the general case, $P=\lim _{\rightarrow} P_{i}$, where the $P_{i}$ are finite spacelike spectra. There is a diagram

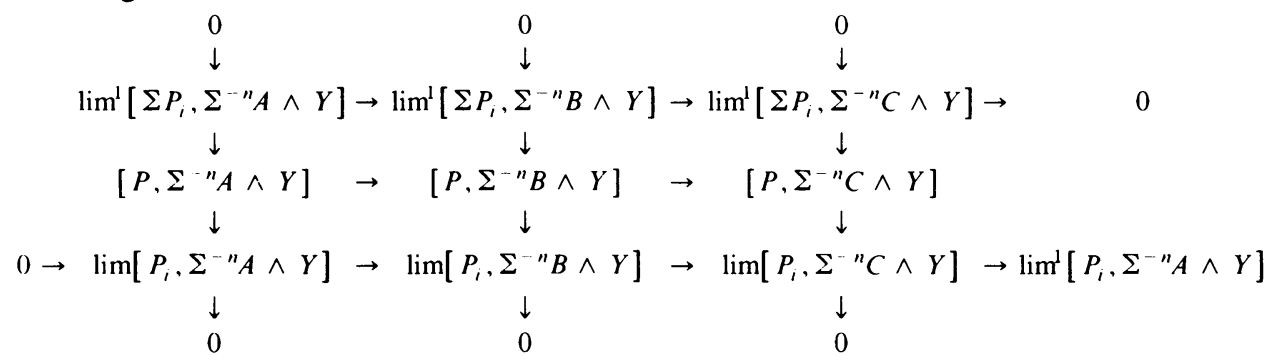


where the vertical sequences are Milnor exact sequences, and the top and bottom ones are part of standard six-term $\lim ^{1}$ exact sequences. Under our assumption about $\pi_{*}(A)$, the sets $\left[\Sigma P_{i}, \Sigma^{-n} A \wedge Y\right]$ and $\left[P_{i}, \Sigma^{-n} A \wedge Y\right]$ are finite. The corresponding $\lim ^{1}$ terms thus vanish, and the exactness of the middle horizontal sequence follows.

3. Examples. Our first applications of Theorem 2.1 are to the exact sequence

$$
\cdots \rightarrow \Sigma L(2) \rightarrow \Sigma L(1) \rightarrow \Sigma L(0) \rightarrow \Sigma H \mathbf{Z}
$$

constructed in our solution to Whitehead's conjecture for $S^{0}$. Here all spectra are to be localized at $p, L(k)=\Sigma^{-k} \mathrm{SP}^{p^{k}} S^{0} / \mathrm{SP}^{p^{k-1}} S^{0}$, and $H \mathbf{Z}$ is the integral EilenbergMac Lane spectrum (which can be identified with $\mathrm{SP}^{\infty} S^{0}$ by the Dold-Thom Theorem). $L(0)=\Sigma^{\infty} S^{0}$ and $L(1)=\Sigma^{\infty} B \Sigma_{p}$, so exactness at $\Sigma L(0)$ is the KahnPriddy Theorem. (The extra suspension is a bit of a bonus.)

For $k \geqslant 1, L(k)$ has finite homotopy groups, since, for example, it is known to be a stable wedge summand of $B(\mathbf{Z} / p)^{k}[12]$. Thus Theorem 2.1 can be directly applied to conclude that, for every finite complex $Y$, there is an exact sequence

$$
\cdots \rightarrow \Sigma^{1-n} L(2) \wedge Y \rightarrow \Sigma^{1-n} L(1) \wedge Y \rightarrow \Sigma^{1-n} \wedge Y \rightarrow \Sigma^{1-n} H \mathbf{Z} \wedge Y
$$

with $n$ chosen so that the $n$-dual of $Y$ is spacelike.

Consideration of the beginning of this sequence yields the next theorem.

THEOREM 3.2. Let $Y$ be a finite complex with $n$ chosen as above. Let

$$
M=\max \{n-1, \operatorname{dim} Y+1\} .
$$

(a) $\pi_{q}^{s}\left(B \Sigma_{p} \wedge Y\right) \rightarrow \pi_{q}^{s}(Y)$ is onto for $q \geqslant M$.

(b) Let $\alpha: \Sigma^{d} Y \rightarrow Y$ be any stable self-map. If $d N \geqslant M$ then the Nth iterate of $\alpha$ lifts:

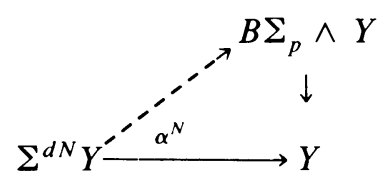

(c) $\Omega^{M} Q\left(B \Sigma_{p} \wedge Y\right) \rightarrow \Omega^{M} Q Y$ is the projection onto a direct factor.

All of these follow immediately from the exactness of (3.1) at $\Sigma^{1-n} Y$ together with the observation that $\Omega^{M} \Omega^{\infty}(H \mathbf{Z} \wedge Y) \simeq *$ since $M>\operatorname{dim} Y$.

Exactness of the whole sequence (3.1) can be used to attack Whitehead's conjectures.

THEOREM 3.3. With $Y$ and $n$ as above, (1.1) is true unless $k+n-1>q \geqslant 2 p^{k+1}$ -2 .

Proof. The key is the observation that, for a spectrum $E, \operatorname{SP}^{m} E=\operatorname{SP}^{m} S^{0} \wedge E$ [11]. Straightforward diagram chasing applied to (3.1) then gives the stated upper bound on $q$. The lower bound is trivial: connectivity arguments show that $\pi_{q}\left(\mathrm{SP}^{p^{h}} Y\right) \rightarrow \pi_{q}\left(\mathrm{SP}^{\infty} Y\right)$ is an isomorphism if $q<2 p^{k+1}-2$. 
EXAMPLES 3.4 .

$$
\begin{aligned}
& \begin{array}{lll}
S^{0} \cup_{2} e^{1} & \underline{n} & (k, q) \text { satisfying the inequalities of Theorem } 3.3 \\
\varnothing
\end{array} \\
& S^{0} \cup_{\eta} e^{2} \quad 4 \quad(0,2) \\
& S^{0} \cup_{\eta^{2}} e^{3} \quad 5 \quad(0,2),(0,3) \\
& S^{0} \cup_{\nu} e^{4} \quad 8 \quad(0, q), \quad 2 \leqslant q<7, \\
& S^{0} \cup_{\sigma} e^{8} \quad 16 \quad(0, q), \quad 2 \leqslant q<15, \\
& (1, q), \quad 6 \leqslant q<16 \text {, } \\
& (2, q), \quad 14 \leqslant q<17
\end{aligned}
$$

When the attaching map is $\eta$ or $\eta^{2}$, easy diagram chasing eliminates the exceptional pairs $(k, q)$. (This includes Welcher's mod 2 examples [11, Corollary 6.5].)

EXAMPLE 3.5. Let $p$ be odd and let $\alpha \in \pi_{2 p-3}\left(S^{0}\right)$ be a generator. Then (1.1) is true for $Y=S^{0} \cup_{\alpha} e^{2 p-2}$. In this case, the inequalities leave open only the cases $(k, q)=(0,2 p-2)$ or $(0,2 p-1)$. Again it is easy to eliminate these cases.

For our next application of Theorem 2.1, we interpret Becker's result about $B O$ as the construction of an onto map $f: \Sigma^{\infty} B O(2) \rightarrow k o\langle 0\rangle$, where $k o\langle 0\rangle$ is the 0 -connected cover of the periodic real $K$-theory spectrum $K O$. Since the fiber of $f$ has finite homotopy groups, Theorem 2.1 applies. Thus letting $Y=S^{0} \cup_{\eta} e^{2}$, there is an onto map

$$
\Sigma^{-4} \Sigma^{\infty} B O(2) \wedge Y \rightarrow \Sigma^{-4} k o\langle 0\rangle \wedge Y .
$$

Now note that $Y=\Sigma^{-2} \mathbf{C} P^{2}, \pi_{q}(k o\langle 0\rangle \wedge Y) \simeq \pi_{q}(K O \wedge Y)$ for $q>2$, and $K O \wedge$ $Y=K U=\Sigma^{-4} K U$ [1, p. 206]. These facts, together with (3.6), imply that there is an onto map $\Sigma^{-6} \Sigma^{\infty} B O(2) \wedge \mathrm{C} P^{2} \rightarrow K U$.

The next theorem follows.

THEOREM 3.7. There is an infinite loop map $\Omega^{6} Q\left(B O(2) \wedge \mathbf{C} P^{2}\right) \rightarrow B U \times \mathbf{Z}$ which is a projection onto a direct summand.

REMARK 3.8. In using Theorem 2.1, it occasionally happens that $n$ can be chosen to be quite small. As an amusing example, let $Y=B(k)$, the $k$ th $\bmod 2$ BrownGitler spectrum with $H^{*}(B(k))=A / A\left\{\chi\left(\mathrm{Sq}^{i}\right) \mid i>k\right\}$. It was conjectured by $\mathrm{H}$. Miller [10] and proved by J. Lannes [8] that the $2 k$ th dual of $B(k)$ is spacelike. Thus we conclude that a short exact sequence of spectra $A \rightarrow B \rightarrow C$ induces a short exact sequence

$$
0 \rightarrow B(k)_{q}(A) \rightarrow B(k)_{q}(B) \rightarrow B(k)_{q}(C) \rightarrow 0
$$

for $q \geqslant 2 k$. Note that this range is essentially complementary to the range of $q$ for which properties of $B(k)$ allow one to do computations.

\section{REFERENCES}

1. J. F. Adams, Stable homotopy and generalized homology, Univ. of Chicago, 1974.

2. J. C. Becker, Characteristic classes and K-theory, Lecture Notes in Math., vol. 428, Springer, 1974, pp. 132-143.

3. J. D. S. Jones, Root invariants and cup-r products in stable homotopy theory, Bull. London Math. Soc. 17 (1985), 479-483. 
4. D. S. Kahn and S. B. Priddy, The transfer and stable homotopy theory, Math. Proc. Cambridge Philos. Soc. 83 (1978), 103-111.

5. N. J. Kuhn, A Kahn-Priddy sequence and a conjecture of $G$. W. Whitehead, Math. Proc. Cambridge Philos. Soc. 92 (1982), 467-483.

6. Spacelike resolutions of spectra, Proc. Northwestern Homotopy Theory Conf., Contemp. Math., vol. 19, Amer. Math. Soc., Providence, R. I., 1983, pp. 153-165.

7. N. J. Kuhn and S. B. Priddy, The transfer and Whitehead's conjecture, Math. Proc. Cambridge Philos. Soc. 98 (1985), 459-480.

8. J. Lannes, Sur le n-dual n-ème spectre de Brown-Gitler, preprint, 1984.

9. R. J. Milgram (Editor), Problems presented to the 1970 Amer. Math. Soc. Summer Colloq. in Algebraic Topology, Algebraic Topology, Proc. Sympos. Pure Math., vol. 22, Amer. Math. Soc., Providence, R. I., 1971, pp. 187-201.

10. H. Miller, The Sullivan conjecture on maps from classifying spaces, Ann. of Math. (2) 120 (1984), 39-87.

11. P. J. Welcher, Symmetric products and the stable Hurewicz homomorphism, Illinois J. Math. 24 (1980), 527-544.

12. S. A. Mitchell and S. B. Priddy, Stable splittings derived from the Steinberg module, Topology 22 (1983), 285-298.

Department of Mathematics, Princeton University, Princeton, New Jersey 08544 